\title{
A Survey for New PN in the Southern Galactic Bulge.
}

\author{
Sylvie F. Beaulieu, Michael A. Dopita、 \\ Kenneth C. Freeman and Agris J. Kalnajs
}

Mount Stromlo and Siding Spring Observatories, Canberra, Australia

We present the results of a deep and uniform narrow-band $\mathrm{H}_{\alpha}$ imaging survey for planetary nebulæ (PNe) in the southern galactic bulge. The goal of this survey was to obtain a sample of bulge tracers which is being used to study the dynamics of the Milky Way bulge (Beaulieu, 1996).

We have chosen PNe as the tracers because they are less affected by metallicity bias than most other tracers and they are strong emitters in $\mathrm{H}_{\alpha}$ - this make their velocities easy to measure.

The $1.0 \mathrm{~m}$ telescope optical imaging survey covers the region of the southern galactic bulge: $1= \pm 20^{\circ}$ and $\mathrm{b}=-5^{\circ}$ to $-10^{\circ}$, chosen because of its lower extinction relative to the northern bulge. In our survey, we have found 56 new PNe and have re-discovered 45 known PNe. We have thus doubled the number of known PNe in our surveyed region.

Using the $1.9 \mathrm{~m}$ telescope we have measured the radial velocities of this uniformly selected sample which is the basis of the comparison with the dynamical models. We have also re-measured radial velocities for a subset of 317 PNe from the Acker al. (1992) Catalogue, in the more extended region $1= \pm 30^{\circ}$ and $b= \pm\left(3.3^{\circ}\right.$ and $\left.15^{\circ}\right)$ : this larger sample is also being used for some dynamical comparisons, although the discovery process for this larger sample is much less homogeneous.

From the COBE/DIRBE 1.25, 2.2 and $3.5 \mu \mathrm{m}$ images, a two-tailed K-S test show that there is no significant difference between the longitude distribution of the PNe and the COBE light in the zone of our deep survey. Also, we find that the extinction in our surveyed fields is not severe and that its distribution is fairly uniform.

\begin{tabular}{|c|c|c|}
\hline & Discovery images & Follow up spectroscopy \\
\hline \hline Observatory & Siding Spring & Mount Stromlo \\
\hline Telescope & $1.0 \mathrm{~m}$ & $1.9 \mathrm{~m}$ \\
\hline Instrument & LDSS & Boller and Chivens spectrograph \\
\hline Detector & $1024 \times 1024 \mathrm{CCD}$ & $385 \times 578 \mathrm{CCD}$ \\
\hline pixel size & $2^{\prime \prime} .35$ & $1 \AA\left(50 \mathrm{~km} \mathrm{~s}^{-1}\right)$ \\
\hline$\lambda$ & $\mathrm{H}_{\alpha}$, Continuum & $\mathrm{H}_{\alpha}$ \\
\hline
\end{tabular}

\section{REFERENCES}

Acker, A. et al. 1992, in The Strasbourg-ESO Catalogue of Galactic Planetary Nebula Part 1 \& 2, (Germany: ESO)

Beaulieu, S.F. 1996, PhD thesis, Australian National University 